\section{When a Silhouette Appears Male: Observer's Own Physical Fitness Governs Social Categorization of Sexually Ambiguous Stimuli}

\author{
Reiki Kishimoto ${ }^{1,2 *}$, Kyoshiro Sasaki ${ }^{2}$, Akihiko \\ Gobara², Yuko Ojiro'2, Giyeon $\mathrm{Nam}^{2}$, Kayo Miura ${ }^{3}$, \\ Yuki Yamada ${ }^{4}$ \\ ${ }^{1}$ Graduate School of Letters, Kyoto University, Yoshida-honmachi, Sakyo- \\ ku, Kyoto 606-8501, Japan \\ ${ }^{2}$ Graduate School of Human-Environment Studies, Kyushu University, \\ 6-19-1, Hakozaki, Higashi-ku, Fukuoka 812-8581, Japan \\ ${ }^{3}$ Faculty of Human-Environment Studies, Kyushu University, 6-19-1, \\ Hakozaki, Higashi-ku, Fukuoka 812-8581, Japan \\ ${ }^{4}$ Faculty of Arts and Science, Kyushu University, 744, Motooka, Nishi-ku, \\ Fukuoka 819-0395, Japan \\ "Author for correspondence (kishimoto.r.k@gmail.com)
}

Categorization bias in social cognition is widely observed, as it is more beneficial to commit low-risk biases in order to reduce high-risk biases. Here, we hypothesized that a low capacity of self-protection leads to a biased social categorization of others as "harmful." To examine this, silhouettes of human body, which had a sexually dimorphic cue (waist-to-hip ratio), were presented, and participants categorized the stimuli as being either "female" or "male." Participants' basic physical fitness was measured, and we manipulated exogenous physical load by either a heavy or light backpack. Physical load did not significantly influence sex categorization. In contrast, only female participants tended to categorize the stimuli as "male" more, as basic physical fitness increased, suggesting that basic physical fitness, not physical load, affects sex categorization for females. Our findings shed new light on the role of fitness factors in social cognition.

\section{Keywords}

sex categorization, error management, basic physical fitness

\section{Introduction}

Every time we encounter strangers, we have to engage in social evaluation by means of various information sources (Fiske \& Neuberg, 1990). Quick and accurate social categorization is an important task for social cognition, because social categories regarding the person in front connote rich and various information. Thus, social categorization provides us with clues of how to interact with that person.

However, social categorization does not always work, and committing categorization errors is inevitable. For example, people sometimes categorize potentially harmful others as safe (false safety bias), and at other times categorize potentially beneficial others as harmful (false danger bias). Even though it is desirable to eliminate both biases completely, given the rapidity, it will be difficult to simultaneously reduce both biases with which we engage in categorical processing. Under these circumstances, the likelihood of one bias will increase with attempts to reduce the other (Haselton \& Nettle, 2006). Accordingly, it is more beneficial to commit low-risk biases in order to reduce high-risk biases. That is, we commit the false danger bias to avoid the false safety bias, and there must be a psychological mechanism that reduces more risky bias at the expense of categorization accuracy. Consequently, social categorization of category members would be biased towards potential harm, and while this strategy would increase overall bias rates, it would minimize the overall risk (Haselton \& Nettle, 2006).

Gender information signalizes the harmfulness of others, with a certain degree of accuracy. Human males are relatively harmful compared to females. For example, homicide was committed predominantly by males all over the world (Hiraiwa-Hasegawa, 2005). Even within males, highly masculine males tend to engage in violence with a greater degree than less masculine males do (Carré \& McCormick, 2008). To sum up, a majority of dangerous individuals are males, and hence, categorizing a stranger as male is adaptive to reduce risks when only sexual dimorphic cue is available. Therefore, it is predicted that sex categorization of sexually ambiguous others should be biased toward males. Consistent with this prediction, previous studies have reported that sex categorization of silhouettes (Johnson, Iida, \& Tassinary, 2012) is biased toward males (i.e., male bias).

When encountering harmful others, people should take protective actions (e.g., escaping from them or defending against their attacks) (Correll, Park, Judd, \& Wittenbrink, 2002). Therefore, it is highly likely that individuals with difficulty in taking such actions, due to poor physical fitness or inability to move freely by some exogenous factors, have more disadvantages in self-protection. Accordingly, social categorization of these individuals should be strongly biased towards as labeling harmful (i.e., male bias in a sex categorization task) for such individuals. In the present study, we investigate this hypothesis by measuring participants' basic physical fitness and imposing physical load on participants. Previous studies revealed that heart rate (HR), saturation of peripheral oxygen $(\mathrm{SpO} 2)$ and body mass index (BMI) have positive correlation with physical activity, energy expenditure or health quality (Eston, Rowlands, \& Ingledew, 1998; Kanai et al., 2014; 
Kajita et al., 1995; Igarashi \& Iijima, 2006). Thus, we used HR, SpO2, and BMI as indices for measuring basic physical fitness. Moreover, physical load was induced by heavy and light backpacks.

\section{Methods}

Participants

Forty-one volunteers (22 females and 19 males, mean age \pm $S D=21.88 \pm 3.36$ years, range $=19-38$ years) participated . Data of one male were excluded from the analysis due to experimental error.

\section{Apparatus and stimuli}

The stimuli were presented on a 46-inch LCD monitor with a resolution of $1920 \times 1080$ pixels and a refresh rate of $60 \mathrm{~Hz}$. The presentation of stimuli and the collection of data were controlled using Mac Pro. Key inputs were acquired using a numerical keypad. Stimuli were presented at a viewing distance of $130 \mathrm{~cm}$ and consisted of a fixation point and images made up of morphed images of female and male silhouettes. The fixation point was composed of two concentric rings: one small and one large, forming a radius of $0.22^{\circ}$ and $0.44^{\circ}$ in visual angle, respectively.

Two computer-generated images were prepared. The waist-to-hip ratios (WHR) of these images were 0.863 and 0.481 . Because WHR is a sexually dimorphic cue (e.g., Johnson et al., 2012), the former and latter stimuli were used as male and female images, respectively. These two stimuli were morphed in order to generate 21 equally stepped morphed images with a percentage of masculinity ranging from $0 \%$ to $100 \%$ (Fig. 1). These 21 morphed bodies varied in WHR. Each stimulus was displayed on a gray background.

\section{Procedure}

Overall design. Participants were told that their task was to wear a backpack for approximately 20 minutes and to complete a dummy questionnaire about its usability. Prior to the experiment, participants' HR and $\mathrm{SpO} 2$ were measured with a fingertip pulse oximeter (Vitel RX-101), and their BMIs were calculated based upon self-reports of body weight and height values. To conceal our real purpose, we told the participants that basic physical fitness might affect the usability evaluation. Upon completion of the experiment, an experimenter asked participants what they thought about the purpose of the experiment was. No participants reported that the two tasks were related to each other.

Each participant was randomly assigned to one of two weight conditions: 20 of them (11 females and 9 males) participated in a heavy condition and 20 (11 females and 9 males) in a light condition. In the heavy condition, each participant wore a backpack with iron dumbbells whose total weights were preset as one seventh of her/his reported body weight throughout the experiment. The procedure of the light condition was identical to that of heavy condition, except that participants merely wore a backpack with no loaded iron dumbbells.

Participants were told to walk along a corridor for about 87 meters while wearing the backpack and to continue the experimental tasks with the same backpack on for about 20 minutes in total. They were also allowed to do anything they wanted except vigorous exercise after the walk. At that time, participants were asked to participate in another experiment regarding sex categorization. To ensure that participants were not aware that we were examining the effect of backpacks on sex categorization, we purposely stated that the following experiment was a part of a different ongoing study in our laboratory. After walking along the corridor, another experimenter entered the room and introduced sex categorization task to participants.

Sex categorization task. Participants were required to remain standing during the course of sex categorization task, which consisted of three sessions: preview, practice, and test. In the preview session, participants merely viewed each silhouette image once in a randomized order to become familiar with the range of stimuli. Then, the participants performed a brief practice. In the test session, each trial consisted of a "start" prompt followed by a randomly selected silhouette image. Participants initiated each trial by pressing the start key. The fixation point was presented throughout the experiment whenever there was no image on the screen. The participants' task was to categorize the morphed image as either of two categories (i.e., female or male) by pressing assigned keys as quickly as possible while maintaining accuracy. A silhouette image remained on the screen until a response was made. The silhouette images were presented in 20 blocks of trials. Each participant performed 420 trials in total (20 repetitions of 21 images).

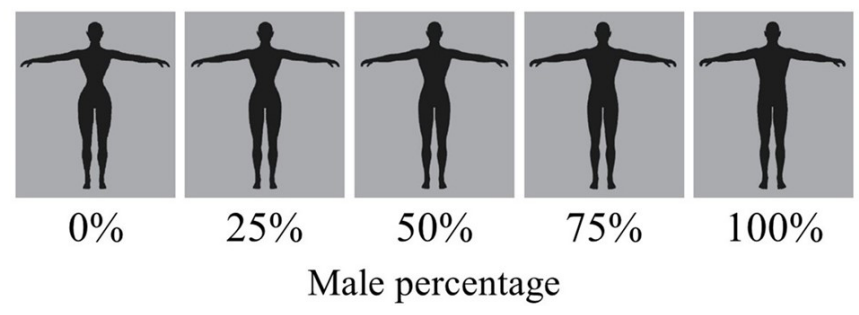

Figure 1. Examples of visual stimuli used in the present study. 


\section{Results}

Subjective boundary on sex categorization

The value of waist breadth / bitrochanteric breadth of each stimulus was defined as WHR. We calculated the point of subjective equality (PSE) for each participant by fitting a cumulative Gaussian function to the proportion of "male" responses as a function of WHR. The data from one female participant were excluded from further analyses because the designated psychometric function failed to fit her data (Deviance $=25.46$ ).

The effect of weight conditions, participants' gender and basic physical fitness

To test whether there were any main effect of weight conditions or participants' gender or interactions between these two factors and basic physical fitness, we performed a hierarchical regression analyses by maximum likelihood estimation with PSE as a criterion variable and with $\mathrm{BMI}, \mathrm{HR}$ and $\mathrm{SpO} 2$ as independent variables with standardization. Table 1 shows the mean values of each index.

First, BMI, HR, and SpO2 were entered on Step 1; the interaction terms between BMI and participants' gender, between HR and participants' gender, and between $\mathrm{SpO} 2$ and participants' gender were added in Step 2; The $R^{2}$ value increased between Steps 1 and $2\left(\Delta R^{2}=.247, p=\right.$ .005 ), indicating that the effect of BMI, HR, and $\mathrm{SpO} 2$ on sex categorization varied dependently on the participants' gender.

To further examine the relationship between participant's gender and other variables, we performed a stepwise regression analysis by maximum likelihood estimation for each gender group (male and female participants). In female participants, $\mathrm{BMI}$ and $\mathrm{SpO} 2$ predicted the degree of male bias $(\beta=.623, p=.003$ and $\beta=.321, p=.012$, respectively; Fig. 2). On the other hand, no variable predicted the degree of male bias in male participants. Furthermore, variances in those three variables were not significantly different between genders (BMI: $F(17,20)=2.581, p=.117$; HR: $F(17,20)=0.116, p$ $=.735 ; \mathrm{SpO} 2: F(20,17)=0.210, p=.649)$.

Second, BMI, HR, and SpO2 were entered on Step 1; the interaction terms between BMI and weight condition, between HR and weight condition, and between $\mathrm{SpO} 2$ and weight condition were added on Step 2; The $R^{2}$ value did not significantly increase between Steps 1 and $2\left(\Delta R^{2}\right.$ $=.039, p=.268)$, indicating that weight conditions had no effect on the degree of male bias and the effect of basic physical fitness on sex categorization did not vary dependently on weight condition.

\section{Discussion}

The aim of the present study was to explore whether poor physical fitness (participants' intrinsic disadvantage) or temporally imposed load (exogenous disadvantage) strengthens the male bias. Our results showed that basic physical fitness negatively predicted male bias at least for female participants, although there are no effect of physical load and no interactions between physical load and basic physical fitness. These findings suggest that the temporal and exogenous states of the body have no influence on sex categorization, while intrinsic factors regarding bodily state are important for sex categorization.

It is possible that each of basic physical fitness and exogenous load affects different stages of threat processing. A recent model of threat processing (Bar-Haim, Lamy, Pergamin, Bakermans-Kranenburg, \& Van Ijzendoorn, 2007) assumes two kinds of threat evaluation system, namely, preattentive and guided threat evaluation systems. The preattentive evaluation system automatically evaluates whether the stimulus in the environment is threatening

Table 1. The mean and $S D$ of each index.

\begin{tabular}{lccc}
\hline Gender & HR & SpO2 & BMI \\
& Mean $\pm S D$ & Mean $\pm S D$ & Mean $\pm S D$ \\
\hline Male $(\mathbf{n}=\mathbf{1 8})$ & $83.00 \pm 13.85$ & $96.61 \pm 1.67$ & $21.16 \pm 3.14$ \\
Female $(\mathbf{n}=\mathbf{2 1})$ & $78.14 \pm 12.48$ & $96.67 \pm 1.96$ & $20.33 \pm 1.79$ \\
Total $(\mathbf{n}=\mathbf{3 9})$ & $80.38 \pm 13.35$ & $96.64 \pm 1.83$ & $20.72 \pm 2.54$ \\
\hline
\end{tabular}

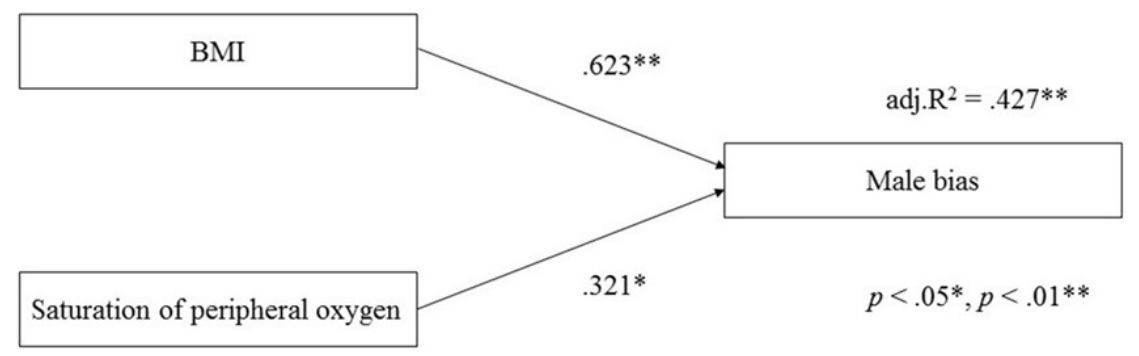

Figure 2. The results of a path analysis for female participants. 
or not. Basic physical fitness cannot be changed easily, and thus, is uncontrollable. Given the present results, this factor might always be closely linked to the categorization of others as harmful or not, and it might be automatically used before the initiation of conscious processing.

During the phase of the guided threat evaluation system, which is the stage that follows the preattentive evaluation system, individuals assess the context of the threatening stimulus, compare it with prior knowledge and memory, and assess the availability of coping recourses. Therefore, because the physical load was a temporally imposed factor - and hence, the context of the threat and the availability of coping resources are assessed in the guided threat evaluation system - the decision made in guided evaluation system might be less dominant than those made in the preattentive evaluation system.

In summary, the present study aimed at clarifying the effect of participants' intrinsic and exogenous disadvantages on sex categorization. We found that basic physical fitness negatively correlated with the degree of male bias at least for female participants, while physical load did not affect sex categorization. Here, we provided two pieces of evidence that low basic physical fitness and high physical load were processed differently and elicited different effects on sex categorization, even though they were disadvantageous in self-protections; and the effect of intrinsic and uncontrollable disadvantages about one's own bodily state is more dominant.

\section{Acknowledgement}

This work was supported by JSPS (\#14J06025, \#26540067, and \#15H05709) and Kyushu University (P\&P and EEP; \#26307 and \#26806). We would like to thank K.Q. and Q.Y. for their insightful comments.

\section{References}

Bar-Haim, Y., Lamy, D., Pergamin, L., BakermansKranenburg, M. J., \& Van Ijzendoorn, M. H. (2007). Threat-related attentional bias in anxious and nonanxious individuals: A meta-analytic study. Psychological Bulletin, 133, 1-24. (doi: 10.1037/00332909.133.1.1)

Carré, J. M., \& McCormick, C. M. (2008). In your face: Facial metrics predict aggressive behaviour in the laboratory and in varsity and professional hockey players. Proceedings of the Royal Society B, 275, 2651-2656. (doi: 10.1098/rspb.2008.0873)

Correll, J., Park, B., Judd, C. M., \& Wittenbrink, B. (2002). The police officer's dilemma: Using ethnicity to disambiguate potentially threatening individuals. Journal of Personality and Social Psychology, 83, 1314-1329. (doi: 10.1037/0022-3514.83.6.1314)

Eston, R. G., Rowlands, A. V., \& Ingledew, D. K. (1998). Validity of heart rate, pedometry, and accelerometry for predicting the energy cost of children's activities. Journal of Applied Physiology, 84, 362-371.

Fiske, S. T., \& Neuberg, S. L., (1990). A continuum model of impression formation from category based to individuating process: Influences of information and motivation on attention an interpretation. In M. P. Zanna,(Ed.), Advances in experimental social psychology (pp. 1-74). New York: Academic Press.

Haselton, M. G., \& Nettle, D. (2006). The paranoid

Kishimoto et al. LEBS Vol. 7 No.1 (2016) 17-20 optimist: An integrative evolutionary model of cognitive biases. Personality and Social Psychology Review, 10, 47-66. (doi: 10.1207/ s15327957pspr1001_3)

Hiraiwa-Hasegawa, M. (2005). Homicide by men in Japan, and its relationship to age, resources and risk taking. Evolution and Human Behavior, 26, 332-343. (doi: 10.1016/j.evolhumbehav.2004.12.003)

Igarashi, H., \& Iijima, S. (2006). Effects of life-style factors and health related factors on subjective health in male workers. Yamanashi Nursing Journal, 4, 1924. (in Japanese)

Johnson, K. L., Iida, M., \& Tassinary, L. G. (2012). Person (mis)perception: Functionally biased sex categorization of bodies. Proceedings of the Royal Society B, 279, 4982-4989. (doi: 10.1098/ rspb.2012.2060)

Kajita, E., Iki, M., Tobita, Y., Mitamura, S., Kusaka, Y., Ogata, A., . . Ishii, Y. (1995). Bone mineral density of the lumbar spine and its relation to biological and lifestyle factors in middle-aged and aged Japanese women (Part 3) Relationships of physical fitness and lifestyle factors to bone mineral density in premenopausal and postmenopausal women. Japanese Journal of Hygiene, 50, 893-900. (doi: 10.1265/ jjh.50.893) (in Japanese)

Kanai, R., Uzawa, K., Moriyama, K., Kotani, M., Kohyama, T., Nakazawa, H., . . . Yorozu T. (2014). Hypoxemia and oxygen saturation by pulse oximeter. Journal of the Japanese Society of Intensive Care Medicine, 21, 175-176. (doi: 10.3918/jsicm.21.175) (in Japanese) 